\title{
Adaptive Analysis of Bifurcation Points of Shell Structures
}

\author{
E. Ewert and K. Schweizerhof \\ Institut für Mechanik, Universität Karlsruhe (TH), \\ Kaiserstraße 12, D-76131 Karlsruhe, Germany \\ mechanik@ifm.uni-karlsruhe.de
}

\section{Introduction}

In recent years many procedures for stability investigations based on the Finite Element Method have been developed to compute the so-called stability points of structures in order to judge the stability. However, in engineering practice predominantly knock-down factors based on experiments are used for the design loads. The reason for such a procedure is the hard to quantify sensitivity of shell structures - especially of cylindrical shells - against perturbations like geometrical and loading imperfections or imperfections in boundary conditions. The standard procedure as also proposed in design rules is based on the computation of the limit load taking into account the modification of the bifurcation load resp. the snap-through load due to geometrical imperfections. Thus, the eigenvalueproblem for stability points have to be computed very accurately. In the present contribution an adaptive $h$-refinement procedure is taken for the solution using low order shell elements. The algorithm is partially based on the well-known a-posteriori error estimator of Zienkiewicz and Zhu [Zie92] with the stresses computed using the eigenvectors instead of displacement vectors, as e.g. proposed by Stein et al. [Stein94].

\section{Static Stability Criteria}

After discretization with finite elements analysis the following nonlinear system of equations must be solved to compute states of equilibrium

$$
\boldsymbol{r}(\boldsymbol{u})-\lambda \boldsymbol{p}=\mathbf{0}
$$

Herein $\boldsymbol{r}$ denotes the vector of internal forces depending nonlinearly on the displacements, $\boldsymbol{p}$ the load vector and $\lambda$ the load multiplier. For a Newton type solution within an incremental iterative procedure, the linearization of system 
of equations (1) leads to

$$
\boldsymbol{K}_{T}\left(\boldsymbol{u}_{i}\right) \Delta \boldsymbol{u}_{i+1}=\lambda \boldsymbol{p}-\boldsymbol{r}\left(\boldsymbol{u}_{i}\right) \quad \text { with } \quad \boldsymbol{u}_{i+1}=\boldsymbol{u}_{i}+\Delta \boldsymbol{u}_{i+1}
$$

Herein $\boldsymbol{K}_{T}$ denotes the tangent stiffness matrix in general nonlinearly depending on the current displacement state and $\Delta \boldsymbol{u}_{i+1}$ is the vector of the displacement increments for the next iteration step. Within the incremental iterative solution process involving an update of the displacement vector the iterations are performed until a certain tolerance is met and convergence is achieved. The tangent stiffness matrix $\boldsymbol{K}_{T}$, which is obtained by linearization of $\boldsymbol{r}(\boldsymbol{u})$, can be used in stability investigations of converged states of equilibrium.

As it is well known for conservative systems as considered here, a state of equilibrium is stable, if $\boldsymbol{K}_{T}$ is positive definite, i.e. all eigenvalues $\mu$ from the standard eigenvalue problem

$$
\left(\boldsymbol{K}_{T}-\mu \boldsymbol{I}\right) \boldsymbol{\Psi}=\mathbf{0}
$$

are larger than zero. Another commonly used stability criterion is based on the determinant of $\boldsymbol{K}_{T}$. As the transition from stable to unstable equilibrium states is characterized by zero eigenvalues $\mu=0$, the characteristic equation of (3) gets

$$
\operatorname{det}\left(\boldsymbol{K}_{T}\right)=0 .
$$

Using e.g. these criteria singular points can be determined.

\section{Computation of Stability Points for a Geometrically Perfect Cylinder Under Axial Compression}

First of all mesh convergence studies are performed for a quarter of a perfect cylinder under axial compression using uniform mesh refinement (Fig. 1). The results achieved with the quarter of a cylinder have been verified for few meshes using a model of the whole cylinder, which required very large memory, see Sect. 4. As boundary conditions the upper and lower edges are hinged allowing displacements in axial direction. At both edges a defined load is applied in axial direction. In this case the analytical solution can be written as

$$
\alpha=\frac{F_{c r}}{F_{c r, c l}}=0.843 \quad \text { with } \quad F_{c r, c l}=\frac{2 \pi E t^{2}}{\sqrt{3\left(1-\nu^{2}\right)}} .
$$

with the normalized critical load $\alpha$ for the given boundary conditions, the critical load $F_{c r}$ and the classical critical load $F_{c r, c l}$, see Yamaki [Yam84]. Two different shell elements are used in the FE model: a bilinear shell element with ANS for the transversal shear strains according to Dvorkin and Bathe [Dvor89] and a biquadratic shell element MITC9 according to Bucalem and Bathe [Buca93]. The results of 4 refinement steps are given in Fig. 2a). The 


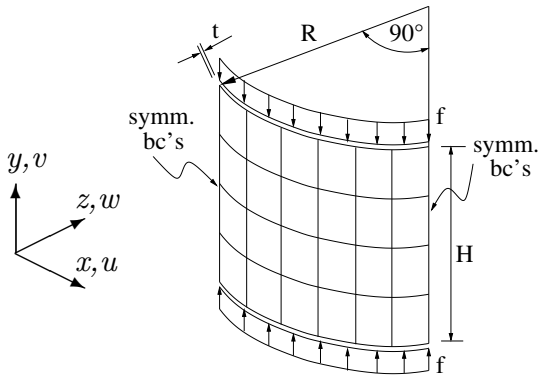

$$
\begin{aligned}
& \text { Geometry: } \\
& \mathrm{R}=625 \mathrm{~mm} \\
& \mathrm{H}=966 \mathrm{~mm} \\
& \mathrm{t}=0.56 \mathrm{~mm} \\
& \text { Material (steel): } \\
& \mathrm{E}=2.0 \cdot 10^{5} \mathrm{~N} / \mathrm{mm}^{2} \\
& \nu=0.3
\end{aligned}
$$

bc's for upper and lower edges:

$\boldsymbol{u}=\boldsymbol{w}=0 ; \boldsymbol{v} \neq 0$

Fig. 1. Quarter of perfect cylinder under axial compression, geometry, material and boundary conditions

a) uniform refinement only

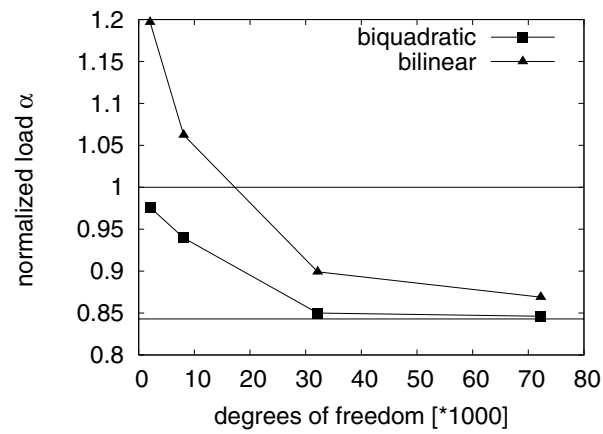

b) uniform and adaptive refinement

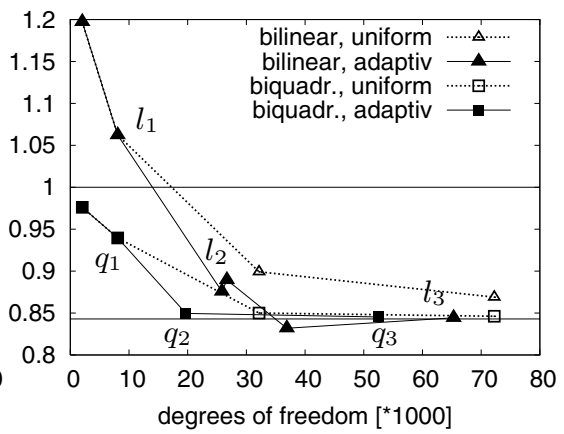

Fig. 2. Static buckling load levels (singular points) of a perfect cylinder modeled with bilinear and biquadratic elements; normalized to classical critical load

convergence diagram for the normalized buckling load $\alpha$ shows a much better convergence for the biquadratic elements than for bilinear elements. Even for a very fine mesh with $120 \times 120$ elements and 72240 degrees of freedom $\left(N_{\text {dof }}\right)$ the deviation of the buckling load computed with bilinear elements from the analytical solution is still about $3 \%$ in this simple example. The reason is the much smoother approximation of the curved geometry using biquadratic elements. Though different eigenvectors are achieved for the starting meshes, both models converge to the same final buckling mode, see Fig. 3 .

An adaptive $h$-refinement procedure might improve the convergence behaviour of a FE solution. In the present contribution a procedure partially based on the well-known a-posteriori error estimator of Zienkiewicz and Zhu [Zie92] is used. The exact error distribution $\|\boldsymbol{e}\|$ is defined by

$$
\|\boldsymbol{e}\|^{2}=\int_{\Omega_{0}}\left(\boldsymbol{\sigma}-\boldsymbol{\sigma}_{h}\right):\left(\boldsymbol{\epsilon}-\boldsymbol{\epsilon}_{h}\right) d \Omega
$$




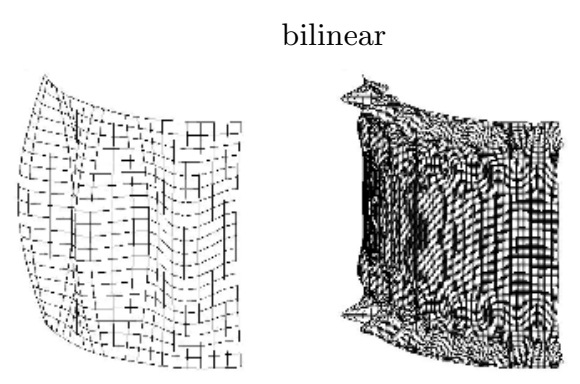

biquadratic
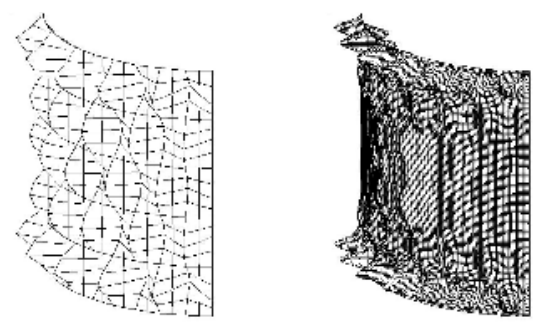

Fig. 3. First eigenvectors at singular points for each coarse $\left(N_{d o f}=2040\right)$ and each fine $\left(N_{d o f}=72240\right)$ meshes; uniform refinement with bilinear and biquadratic elements

with exact values of stresses $\boldsymbol{\sigma}$ resp. strains $\boldsymbol{\epsilon}$. The stresses $\boldsymbol{\sigma}_{h}$ resp. strains $\boldsymbol{\epsilon}_{h}$ are the discrete values the FE solution. As the exact values are unknown the expression (6) is approximated by

$$
\left\|\boldsymbol{e}_{h}\right\|^{2}=\int_{\Omega_{0}}\left(\boldsymbol{\sigma}^{*}-\boldsymbol{\sigma}_{h}\right):\left(\boldsymbol{\epsilon}^{*}-\boldsymbol{\epsilon}_{h}\right) d \Omega .
$$

The values denoted with "*" are called recovered values and are computed by a least-square fit on element patches using the so-called superconvergent patch recovery procedure, see Zienkiewicz and Zhu [Zie92]. Using the recovered and the discrete values the error distribution can be computed and used for mesh refinement. The values resp. the error distribution is computed here using the lowest eigenvector of system of equations (3) at the singular point, similar to the proposal of Stein et al. [Stein94].

The following procedure is followed in an adaptive computation of singular points and buckling modes: starting with an initial mesh, e.g. $20 \times 20$ elements, singular points are computed using a nonlinear computation monitoring the eigenvalues of interest in system of equations (3) in combination with the bisection procedure. The first eigenvalue at this point is then obtained using the standard eigenvalue problem (3). Finally a refined mesh is achieved using the error distribution, which is computed as described before. Then the next refinement step is performed for the refined mesh starting with the computation of a new singular point. The results of an adaptive analysis using bilinear 
elements show, that the convergence behaviour concerning the singular point could be improved, see Fig. 2b). In contrast to this the found buckling modes do not converge to the target mode of the uniformly refined mesh, see Fig. 4. This is due to transition elements, which appear in adaptive meshes and are often considerably distorted for curved shells compared to standard element form. Therefore they often cause artificial geometrical imperfections leading to buckling modes, which are partially totally different from the correct modes. Modeling the cylinder with biquadratic elements these imperfections introduced by imperfect geometry approximation are small enough and do not influence the computation of eigenmodes at singular points distinctly, see Fig. 5.

The sensitivity concerning stability depends strongly on the choice of the perturbation patterns, see Ewert et al. [Ewe05]. As the perturbation patterns are often chosen affine to the eigenvectors, the latter should be computed as accurately as possible for following sensitivity investigations.

$l_{1}$

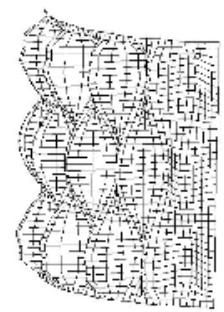

$l_{2}$

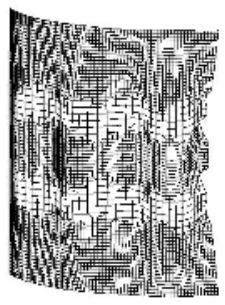

$l_{3}$

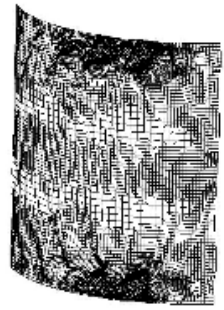

Fig. 4. First eigenvectors at singular points for refinement steps $l_{1}\left(N_{\text {dof }}=8080\right)$, $l_{2}\left(N_{d o f}=25834\right)$ and $l_{3}\left(N_{d o f}=65315\right)$ indicated in Fig. 2 ; adaptive refinement using bilinear elements

$q_{1}$

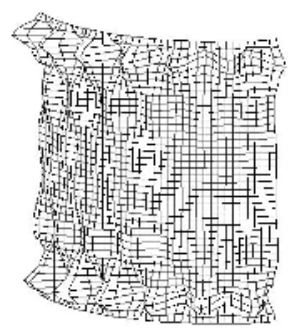

$q_{2}$

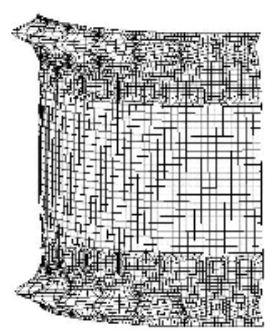

$q_{3}$

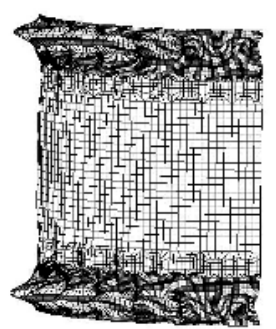

Fig. 5. First eigenvectors at singular points for refinement steps $q_{1}\left(N_{\text {dof }}=8080\right)$, $q_{2}\left(N_{d o f}=19698\right)$ and $q_{3}\left(N_{d o f}=52442\right)$ indicated in Fig. 2 ; adaptive refinement using biquadratic elements 


\section{Computational Aspects}

For the investigations described above the own FE-code named FEAP-MeKA is used. This code is written in FORTRAN 77 (compiled with the Intel compiler) and is based on the open-source FE-code named FEAP (Finite Element Ananlysis Program) from the University of Berkeley.

Below some examples for computational time and memory requirement of calculations performed on a single processor are given.

- Bisection for a quarter of a perfect cylinder with 72000 dof $(120 \times 120$ bilin. el.):

- comp. time: $5 \mathrm{~h} 6 \mathrm{~m} 23 \mathrm{~s}$ (accuracy $1 \cdot 10^{-5}$ )

- comp. time: $2 \mathrm{~h} 15 \mathrm{~m} 11 \mathrm{~s} \mathrm{(accuracy} 1 \cdot 10^{-2}$ )

- physical memory: $668 \mathrm{MB}$

- virtual memory: $5488 \mathrm{MB}$

- Direct computation of singular point for a whole perfect cylinder with 71761 dof $(60 \times 240$ biquad. el. $)$ :

- comp. time: $21 \mathrm{~m} 17 \mathrm{~s}$ (accuracy $1 \cdot 10^{-5}$ )

- physical memory: $2001 \mathrm{MB}$

- virtual memory: $5488 \mathrm{MB}$

- Bisection for a whole perfect cylinder with 287521 dof $(120 \times 480$ bilin. el.)

- comp. time: more than 3 days

- comp. time for a single iteration: $28 \mathrm{~m} 9 \mathrm{~s}$

- physical memory: $5289 \mathrm{MB}$

- virtual memory: $5488 \mathrm{MB}$

Investigations with the parallelized version of FEAP-MeKA lead to the speedup diagram in Fig. 6, which is typical for the investigated problems.

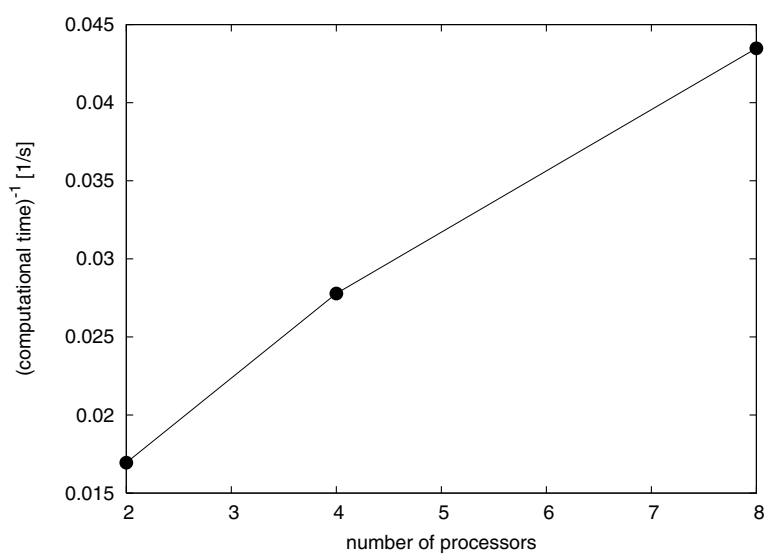

Fig. 6. Speed-up diagramm for the parallelized FEAP-MeKA; typical run for buckling analysis 


\section{References}

[Buca93] Bucalem ML, Bathe KJ.: Higher-order MITC general shell elements. Int. J. Num. Meth. Eng. 36: 3729-3754 (1993)

[Dvor89] Dvorkin EN, Bathe KJ: A continuum mechanics based four node shell element for general nonlinear analysis. Eng. Comp.1: 77-88 (1989)

[Yam84] Yamaki N.: Elastic stability of circular cylindrical shells. North-Holland series in applied mathematics and mechanics, 27, (1984)

[Zie92] Zienkiewicz O and Zhu J.: The superconvergent patch recovery and a posteriori error estimates. Part 1: The recovery technique. Part 2: Error estimates and adaptivity. Int. J. Num. Meth. Eng. 33: 1131-1382 (1992)

[Ewe05] Ewert E, Schweizerhof K, Vielsack P.: Measures to judge the sensitivity of thin-walled shells concerning stability under different loading conditions. Int. J. Comp. Mech. 37: 507-522 (2006)

[Stein94] Stein, E, Seifert, B, Ohnimus, S, Carstensen, C. Adaptive Finite Element Analysis of Geometrically Non-linear Plates and Shells, Especially Buckling. Int. J. Num. Meth. Eng., 37-15: 2631-2656 (1994) 